PROCEEDINGS OF THE

AMERICAN MATHEMATICAL SOCIETY

Volume 134, Number 12, December 2006, Pages 3495-3505

S 0002-9939(06)08720-X

Article electronically published on June 27, 2006

\title{
GREEDY APPROXIMATION WITH RESPECT TO CERTAIN SUBSYSTEMS OF THE WALSH ORTHONORMAL SYSTEM
}

\author{
MARTIN G. GRIGORIAN AND ROBERT E. ZINK
}

(Communicated by Michael T. Lacey)

\begin{abstract}
In an article that appeared in 1967, J.J. Price has shown that there is a vast family of subsystems of the Walsh orthonormal system each of which is complete on sets of large measure. In the present work it is shown that the greedy algorithm, when applied to functions in $L^{1}[0,1]$, is surprisingly effective for these nearly-complete families. Indeed, if $\Phi$ is such a subsystem of the Walsh system, then to each positive $\varepsilon$, however small, there corresponds a Lebesgue measurable set $E$ such that for every $f$, Lebesgue integrable on $[0,1]$, the greedy approximants to $f$, associated with $\Phi$, converge, in the $L^{1}$ norm, to an integrable function $g$ that coincides with $f$ on $E$.
\end{abstract}

Because this simple theoretical algorithm is a model for a procedure widely used in numerical applications, the greedy algorithm has been extensively studied by many investigators.

Of particular interest are some results, connected with the trigonometric system, due to Temlyakov [5], who has demonstrated the existence of functions in $L^{p}[0,1], 1 \leq p<2$, for which a sequence of corresponding greedy approximants diverges in measure, and to Körner [3], who has constructed a continuous function whose Fourier sum, when taken in decreasing order of magnitude, diverges unboundedly almost everywhere.

In connection with these results, one might ask whether it always be possible to alter the values of a given function, on a small set, so that the greedy algorithm, when applied to the function thus altered, will yield a sequence of approximants that does, in some sense, converge. This question has been answered, in the affirmative, by Grigorian [1, who has shown that for each integrable function a modification of this type will always lead to an approximating sequence that converges to the altered function in the $L^{1}$ norm.

Since the trigonometric and Walsh systems have many properties in common, one would think that there should be a corresponding result for the Walsh system. This is, indeed, the case, and, in fact, the same sort of result holds for a multitude of Walsh subsystems, many of them quite sparse and far from complete.

Received by the editors May 10, 2005.

2000 Mathematics Subject Classification. Primary 42C10.

(C)2006 American Mathematical Society 
The Walsh system, an extension of the Rademacher system, may be obtained in the following manner.

Let $r$ be the periodic function, of least period 1 , defined on $[0,1)$ by

$$
r=\chi_{\left[0, \frac{1}{2}\right)}-\chi_{\left[\frac{1}{2}, 1\right)} .
$$

The Rademacher system, $\mathcal{R}=\left\{r_{n}: n=0,1, \ldots\right\}$, is defined by the conditions

$$
r_{n}(x)=r\left(2^{n} x\right), \forall x \in \mathbb{R}, n=0,1, \ldots,
$$

and, in the ordering employed by Payley, the $n$th element of the Walsh system, $\mathcal{W}=\left\{W_{n}: n=0,1, \ldots\right\}$, is given by

$$
W_{n}=\prod_{k=0}^{\infty} r_{k}^{n_{k}},
$$

where $\sum_{k=0}^{\infty} n_{k} 2^{k}$ is the unique binary expansion of $n$, with each $n_{k}$ either 0 or 1 .

The subsystems of $\mathcal{W}$ introduced by Price [4] are defined with the assistance of the following notion of asymptotic density of a sequence of natural numbers.

Definition. Let $S=\left\{n_{j}\right\}_{j=1}^{\infty}$ be an increasing sequence of natural numbers, let $\Delta(n)$ be the number of elements of $S$ that are less than $n$, and let

$$
\rho(\delta)=\limsup _{k} \limsup _{n} \frac{\Delta(n+k)-\Delta(n)}{k} .
$$

Theorem A (Price). Let $S=\left\{n_{j}\right\}_{j=1}^{\infty}$ be an increasing sequence of natural numbers, and let $\mathcal{W}_{s}=\left\{W_{n_{j}}: j \in \mathbb{N}\right\}$. If $\rho(S)=1$, then, for every $\varepsilon>0$, there exists a measurable set $E_{\varepsilon} \subset[0,1]$ such that $\left|E_{\varepsilon}\right|>1-\varepsilon$, and $\mathcal{W}_{s}$ is complete in $L^{2}\left(E_{\varepsilon}\right)$.

The current study is concerned with these nearly-complete subsystems of the Walsh system. The approximations to integrable functions, considered in the sequel, are drawn from the span of a system of this kind.

Definition. Let $\Phi=\left\{\varphi_{n}: n=1,2, \ldots\right\}$ be a subsystem of the Walsh system, let $f \in L^{1}[0,1]$, and, for each $n$, let

$$
c_{n}=c_{n}(f)=\int_{0}^{1} f \varphi_{n} d t
$$

If $m \in \mathbb{N}$ and if $A_{m} \subset \mathbb{N}$ is a set of cardinality $m$ for which

$$
\left|c_{k}\right| \geq\left|c_{j}\right|, \forall k \in A_{m}, \forall j \notin A_{m},
$$

then

$$
\sum_{k \in A_{m}} c_{k} \varphi_{k}
$$

is termed a greedy approximant of $f$ with respect to the family $\Phi$.

Theorem 1. Let $S=\left\{n_{j}\right\}_{j=1}^{\infty}$ be an increasing sequence of natural numbers with $\rho(S)=1$, and let $\varepsilon$ be an arbitrary positive number. There exists a measurable set $E_{\varepsilon} \subset[0,1]$, with $\left|E_{\varepsilon}\right|>1-\varepsilon$, such that to every $f \in L^{1}[0,1]$ there corresponds an integrable function $g$, that coincides with $f$ on $E_{\varepsilon}$, whose $\mathcal{W}_{S}-$ Fourier sum, when taken in decreasing order of magnitude, of the expansion coefficients, converges to $g$ in the $L^{1}$ norm. 
Although a direct proof for this theorem could be given, a more interesting program yields this result as a corollary of the following proposition, which demonstrates the existence of a universal sequence of Walsh polynomials, subsequences of which yield convergent series that approximate the integrable functions arbitrarily closely.

Theorem 2. Let $\mathcal{W}_{S}=\left\{\varphi_{k}: k \in \mathbb{N}\right\}$ be the Walsh subsystem that corresponds to $S$, an increasing sequence of natural numbers for which $\rho(S)=1$. For each $\varepsilon>0$, there are a measurable set $E \subset[0,1]$, with $|E|>1-\varepsilon$, a monotonically decreasing sequence of positive numbers $\left\{a_{k}\right\}_{k=1}^{\infty}$, and an injective function $\sigma: \mathbb{N} \rightarrow \mathbb{N}$ such that to each $f \in L^{1}[0,1]$ there correspond a $g \in L^{1}[0,1]$ and a sequence $\left\{\varepsilon_{k}\right\}_{k=1}^{\infty}$, with each $\varepsilon_{k}$ either 0 or 1 , such that $g$ coincides with $f$ on $E$,

$$
g \stackrel{L^{1}}{=} \sum_{k=1}^{\infty} \varepsilon_{k} a_{k} \varphi_{\sigma(k)}
$$

and

$$
\varepsilon_{k} a_{k}=\int_{0}^{1} g \varphi_{\sigma(k)} d t
$$

The proof of Theorem 2 depends upon a sequence of lemmata, the first of which is one part of Lemma 2 taken from the article [2].

Lemma B. Let $S=\left\{n_{k}\right\}_{k=1}^{\infty}$ be an increasing sequence of natural numbers, with $\rho(S)=1$, and let $\mathcal{W}_{S}$ be the corresponding Price-Walsh system. If $\Delta_{0}$ is an interval of the form $\left[\frac{i-1}{2^{m}}, \frac{i}{2^{n}}\right]$, for some $m \in \mathbb{N}$ and $1 \leq i \leq 2^{m}$, if $k_{0}$ is a natural number greater than 1 , and if the real numbers $\varepsilon$ and $\gamma$ satisfy the conditions $0<\varepsilon<1$ and $\gamma \neq 0$, then there exist a measurable set $E \subset \Delta_{0}$, and a Walsh polynomial

$$
Q=\sum_{k=k_{0}}^{K} c_{n_{k}} W_{n_{k}} \in \operatorname{Span\mathcal {W}}_{S}
$$

such that

$$
\begin{gathered}
Q(x)= \begin{cases}\gamma, & \text { if } x \in E, \\
0, & \text { if } x \notin \Delta_{0},\end{cases} \\
|E|>(1-\varepsilon)\left|\Delta_{0}\right|, \\
\int_{0}^{1}|Q| d t \leq 2|\gamma|\left|\Delta_{0}\right|,
\end{gathered}
$$

and

$$
\max \left\{\left[\int_{0}^{1}\left|\sum_{k=k_{0}}^{m} c_{n_{k}} W_{n_{k}}\right|^{2} d t\right]^{1 / 2}: k_{0} \leq m \leq k\right\} \leq A|\gamma| \varepsilon^{-\frac{1}{2}}\left|\Delta_{0}\right|^{\frac{1}{2}},
$$

where $A$ is an absolute constant.

Lemma 3. Let $S=\left\{n_{k}\right\}_{k=1}^{\infty}$ be an increasing sequence of natural numbers, with $\rho(S)=1$, and let $\Phi=\left\{\varphi_{k}: k \in \mathbb{N}\right\}$ be the Walsh subsystem corresponding to $S$. If $\Delta=\left[\frac{i-1}{2^{m}}, \frac{i}{2^{m}}\right]$, for some $m \in \mathbb{N}$ and $1 \leq i \leq 2^{m}$, if $N_{0}$ is a natural number, and 
if the real numbers $\delta, \varepsilon$, and $\gamma$ satisfy the conditions $\delta>0,0<\varepsilon<1$, and $\gamma \neq 0$, then there exist a measurable set $E \subset \Delta$ and a Walsh polynomial

$$
Q=\sum_{k=N_{0}+1}^{N} a_{k} \varphi_{\sigma(k)}
$$

where $\sigma$ is a permutation of an integer segment $\left\{N_{0}+1, N_{0}+2, \ldots, \bar{N}\right\}$, with $\bar{N}>N$, such that

$$
\begin{aligned}
& Q(x)= \begin{cases}\gamma, & \text { if } x \in E, \\
0, & \text { if } x \notin \Delta,\end{cases} \\
& \int_{0}^{1}|Q| d t \leq 2|\gamma||\Delta|, \\
& |E|>(1-\varepsilon)|\Delta|, \\
& \delta>\left|a_{k}\right| \geq\left|a_{k+1}\right|>0, \quad \forall k \in\left[N_{0}, N\right),
\end{aligned}
$$

and

$$
\max \left\{\int_{0}^{1}\left|\sum_{k=N_{0}}^{m} a_{k} \varphi_{\sigma(k)}\right| d t: N_{0}<m \leq N\right\} \leq A|\gamma| \varepsilon^{-\frac{1}{2}}|\Delta|^{\frac{1}{2}}
$$

where $A$ is the constant from Lemma $\mathrm{B}$.

Proof. Choose $t \in \mathbb{N}$ such that $2^{-t} A|\gamma| \varepsilon^{-\frac{1}{2}}|\Delta|<\delta$, and let $\left\{\Delta_{1}, \Delta_{2}, \ldots, \Delta_{2^{t}}\right\}$ be a partition of $\Delta$ into congruent, dyadic subintervals. One may employ Lemma $\mathrm{B}$ in order to find a sequence of natural numbers

$$
N_{0}=\bar{N}_{0}<\bar{N}_{1}<\bar{N}_{2}<\ldots<\bar{N}_{2^{t}}
$$

a corresponding sequence of Walsh polynomials

$$
Q_{j}=\sum_{k=\bar{N}_{j-1}+1}^{\bar{N}_{j}} b_{k} \varphi_{k}, j=1, \ldots, 2^{t},
$$

and measurable sets $E_{j} \subset \Delta_{j}, j=1, \ldots, 2^{t}$, such that

$$
\begin{aligned}
& Q_{j}(x)= \begin{cases}\gamma, & \text { if } x \in E_{j}, \\
0, & \text { if } x \notin \Delta_{j},\end{cases} \\
& \left|E_{j}\right|>(1-\varepsilon)\left|\Delta_{j}\right|, \\
& \int_{0}^{1}\left|Q_{j}\right| d t \leq 2|\gamma|\left|\Delta_{j}\right|,
\end{aligned}
$$

and

$$
\max \left\{\left(\int_{0}^{1}\left|\sum_{k=\bar{N}_{j-1}+1}^{m} b_{k} \varphi_{k}\right|^{2} d t\right)^{1 / 2}: \bar{N}_{j-1}+1 \leq m \leq \bar{N}_{j}\right\} \leq A|\gamma| \varepsilon^{-\frac{1}{2}}\left|\Delta_{j}\right|^{\frac{1}{2}}
$$

Since

$\max \left\{\left(\int_{0}^{1}\left|\sum_{k=\bar{N}_{j-1}+1}^{m} b_{k} \varphi_{k}\right|^{2} d t\right)^{1 / 2}: \bar{N}_{j-1}<m \leq \bar{N}_{j}\right\}=\left(\sum_{k=\bar{N}_{j-1}+1}^{\bar{N}_{j}} b_{k}^{2}\right)^{1 / 2}$, 
one has

$$
\left|b_{k}\right| \leq A|\gamma| \varepsilon^{-\frac{1}{2}}\left|\Delta_{j}\right|^{\frac{1}{2}}<\delta, \forall k \in \bigcup_{j=1}^{2^{t}}\left(\bar{N}_{j-1}, \bar{N}_{j}\right) .
$$

Let $\sigma$ be a permutation of the segment $\left\{N_{0}+1, \ldots, \bar{N}_{2^{t}}\right\}$ such that

$$
\left|b_{\sigma(k)}\right| \geq\left|b_{\sigma(k+1)}\right|, \quad \forall k \in\left(N_{0}, \bar{N}_{2^{t}}\right),
$$

and let $N=\max \left\{k:\left|b_{\sigma(k)}\right|>0\right\}$.

For each $k \in\left(N_{0}, N\right]$, let

$$
a_{k}=b_{\sigma(k)},
$$

let

$$
Q=\sum_{k=N_{0}+1}^{N} a_{k} \varphi_{\sigma(k)},
$$

and let

$$
E=\bigcup_{j=1}^{2^{t}} E_{j}
$$

Then

$$
\begin{gathered}
Q(x)= \begin{cases}\gamma, & \text { if } x \in E, \\
0, & \text { if } x \notin \Delta,\end{cases} \\
|E|>(1-\varepsilon)|\Delta|, \\
\delta>\left|a_{k}\right| \geq\left|a_{k+1}\right|>0, \forall k \in\left(N_{0}, N\right),
\end{gathered}
$$

and, finally,

$$
\begin{aligned}
& \max \left\{\int_{0}^{1}\left|\sum_{k=N_{0}+1}^{m} a_{k} \varphi_{\sigma(k)}\right| d t: N_{0}<m \leq N\right\} \\
& \leq \max \left\{\left(\int_{0}^{1}\left|\sum_{k=N_{0}+1}^{m} a_{k} \varphi_{\sigma(k)}\right|^{2} d t\right)^{1 / 2}: N_{0}<m \leq N\right\} \\
& =\left(\sum_{k=N_{0}+1}^{N} a_{k}^{2}\right)^{1 / 2} \\
& =\left(\sum_{j=1}^{2^{t}} \sum_{k=\bar{N}_{j-1}+1} b_{k}^{2}\right)^{1 / 2} \\
& \leq\left(\sum_{j=1}^{2^{t}} A^{2}|\gamma|^{2} \varepsilon^{-1}\left|\Delta_{j}\right|\right)^{1 / 2} \\
& \leq\left(A^{2}|\gamma|^{2} \varepsilon^{-1}|\Delta|\right)^{1 / 2} \\
& =A|\gamma| \varepsilon^{-\frac{1}{2}}|\Delta|^{\frac{1}{2}} .
\end{aligned}
$$


Lemma 4. Let $f$ be a (dyadic) step function on $[0,1]$,

$$
f=\sum_{i=1}^{2^{n}} \gamma_{i} \chi_{\Delta_{i}}
$$

where

$$
\Delta_{i}=\left(\frac{i-1}{2^{n}}, \frac{i}{2^{n}}\right), i=1, \ldots, 2^{n},
$$

and let $\Phi=\left\{\varphi_{k}: k \in \mathbb{N}\right\}$ be a subsystem of the Walsh system that satisfies the conditions of Theorem 1. Then, for each $\varepsilon \in(0,1), \delta>0$, and $N_{0} \in \mathbb{N}$, there are a measurable set $E \subset[0,1]$ and a Walsh polynomial

$$
Q=\sum_{k=N_{0}+1}^{N} a_{k} \varphi_{\sigma(k)},
$$

where $\sigma$ is a permutation of the set $\left\{N_{0}+1, \ldots, N, N+1, \ldots, \bar{N}\right\}$, which satisfy the following conditions:

$$
\begin{gathered}
|E|>1-\varepsilon, \\
Q(x)=f(x), \quad \forall x \in E, \\
\int_{0}^{1}|Q| d t \leq 2 \int_{0}^{1}|f| d t, \\
\delta>\left|a_{k}\right| \geq\left|a_{k+1}\right|>0, \quad \forall k \in\left(N_{0}, N\right),
\end{gathered}
$$

and

$$
\max \left\{\int_{0}^{1}\left|\sum_{k=N_{0}+1}^{m} a_{k} \varphi_{\sigma(k)}\right| d t: N_{0}<m \leq N\right\}<3 \int_{0}^{1}|f| d t .
$$

Proof. Since the partition of $[0,1]$ on which the values of $f$ are defined could be dyadically refined, one may assume that

$$
A\left|\gamma_{0}\right|\left|\Delta_{i}\right|^{\frac{1}{2}}<\varepsilon^{\frac{1}{2}} \int_{0}^{1}|f| d t, \forall i \in\left[1,2^{n}\right],
$$

where $A$ is the constant given above.

By virtue of Lemma 3, there are integers $N_{1}$ and $\bar{N}_{1}$ with $N_{0}<N_{1} \leq \bar{N}_{1}$, a measurable set $E_{1} \subset \Delta_{1}$, and a Walsh polynomial

$$
Q_{1}=\sum_{k=N_{0}+1}^{N_{1}} a_{k}^{(1)} \varphi_{\sigma_{1}(k)}
$$

where $\sigma_{1}$ is a permutation of $\left\{N_{0}+1, \ldots, \bar{N}_{1}\right\}$, such that

$$
\begin{aligned}
& Q_{1}(x)= \begin{cases}\gamma_{1}, & \text { if } x \in E_{1}, \\
0, & \text { if } x \notin \Delta_{1},\end{cases} \\
& \left|E_{1}\right|>(1-\varepsilon)\left|\Delta_{1}\right|, \\
& \delta>\left|a_{k}^{(1)}\right| \geq\left|a_{k+1}^{(1)}\right|>0, \forall k \in\left(N_{0}, N_{1}\right), \text { and } a_{k}^{(1)}=0, \forall k>N_{1}, \\
& \int_{0}^{1}\left|Q_{1}\right| d t \leq 2\left|\gamma_{1}\right|\left|\Delta_{1}\right|,
\end{aligned}
$$


and

$$
\max \left\{\int_{0}^{1}\left|\sum_{k=N_{0}+1}^{m} a_{k}^{(1)} \varphi_{\sigma(k)}\right| d t: N_{0}<m \leq N_{1}\right\} \leq A\left|\gamma_{1}\right| \varepsilon^{-\frac{1}{2}}\left|\Delta_{1}\right|^{\frac{1}{2}} .
$$

Similarly, there are natural numbers $N_{2}$ and $\bar{N}_{2}$, with $\bar{N}_{1}<N_{2} \leq \bar{N}_{2}$, a measurable set $E_{2} \subset \Delta_{2}$, and a Walsh polynomial

$$
Q_{2}=\sum_{k=\bar{N}_{1}+1}^{N_{2}} a_{k}^{(2)} \varphi_{\sigma_{2}(k)}
$$

where $\sigma_{2}$ is a permutation of $\left\{\bar{N}_{1}+1, \ldots, \bar{N}_{2}\right\}$, such that

$$
\begin{aligned}
& Q_{2}(x)= \begin{cases}\gamma_{2}, & \text { if } x \in E_{2}, \\
0, & \text { if } x \notin \Delta_{2},\end{cases} \\
& \left|E_{2}\right|>(1-\varepsilon)\left|\Delta_{2}\right|, \\
& \int_{0}^{1}\left|Q_{2}\right| d t \leq 2\left|\gamma_{2}\right|\left|\Delta_{2}\right|, \\
& \left|a_{N_{1}}^{(1)}\right|>\left|a_{k}^{(2)}\right| \geq\left|a_{k+1}^{(2)}\right|>0, \quad \forall k \in\left(\bar{N}_{1}, N_{2}\right),
\end{aligned}
$$

and

$$
\max \left\{\int_{0}^{1}\left|\sum_{k=\bar{N}_{1}+1}^{m} a_{k}^{(2)} \varphi_{\sigma(k)}\right| d t: \bar{N}_{1}<m \leq N_{2}\right\} \leq A\left|\gamma_{2}\right| \varepsilon^{-\frac{1}{2}}\left|\Delta_{2}\right|^{\frac{1}{2}} .
$$

Proceeding thus, inductively, one determines sequences of natural numbers $\left\{\bar{N}_{i}\right\}_{i=1}^{2^{n}}$ and $\left\{N_{i}\right\}_{i=1}^{2^{n}}$, with

$$
N_{0}<N_{1} \leq \bar{N}_{1}<N_{2} \leq \bar{N}_{2}<\ldots<N_{2^{n}} \leq \bar{N}_{2^{n}}
$$

and, for each $i \in\left[1,2^{n}\right]$, a measurable set $E_{i} \subset \Delta_{i}$ and a Walsh polynomial

$$
Q_{i}=\sum_{k=\bar{N}_{i-1}+1}^{N_{i}} a_{k}^{(i)} \varphi_{\sigma_{i}(k)}
$$

where $\sigma_{i}$ is a permutation of $\left\{\bar{N}_{i-1}+1, \ldots, \bar{N}_{i}\right\}$, such that

$$
\begin{aligned}
& Q_{i}(x)= \begin{cases}\gamma_{i}, & \text { if } x \in E_{i}, \\
0, & \text { if } x \notin \Delta_{i},\end{cases} \\
& \left|E_{i}\right|>(1-\varepsilon)\left|\Delta_{i}\right|, \\
& \int_{0}^{1}\left|Q_{i}\right| d t \leq 2\left|\gamma_{i}\right|\left|\Delta_{i}\right|, \\
& \max \left\{\int_{0}^{1}\left|\sum_{k=\bar{N}_{i-1}+1}^{m} a_{k}^{(i)} \varphi_{\sigma_{i}(k)}\right| d t: \bar{N}_{i-1}<m \leq N_{i}\right\} \leq A\left|\gamma_{i}\right| \varepsilon^{-\frac{1}{2}}\left|\Delta_{i}\right|^{\frac{1}{2}},
\end{aligned}
$$

and

$$
\left|a_{N_{i}}^{(i)}\right|>\left|a_{k}^{(i+1)}\right| \geq\left|a_{k+1}^{(i+1)}\right|>0, \forall k \in\left(\bar{N}_{i}, N_{i+1}\right), i=1, \ldots, 2^{n}-1 .
$$

Let $\bar{N}_{0}=N_{0}$, let $N=N_{0}+\sum_{i=1}^{2^{n}}\left(N_{i}-\bar{N}_{i-1}\right)$, and let the sequences $\{\sigma(k)\}$ and $\left\{a_{k}\right\}$ be defined on the integer segment $\left\{N_{0}+1, \ldots, N\right\}$ in the following manner. 
Let $t_{0}=N_{0}=\bar{N}_{0}$, and, for each $j, 1 \leq j \leq 2^{n}$, let $t_{j}=\sum_{i=0}^{j} N_{i}-\sum_{i=0}^{j-1} \bar{N}_{i}$. Then, for $t_{j-1}<k \leq t_{j}$, and $j=1,2, \ldots$, let

$$
\sigma(k)=\sigma_{j}\left(k-t_{j-1}+\bar{N}_{j-1}\right)
$$

and

$$
a_{k}=a_{k-t_{j-1}+\bar{N}_{j-1}}^{(j)} .
$$

Let

$$
E=\bigcup_{i=1}^{2^{n}} E_{i}
$$

and let

$$
Q=\sum_{i=1}^{2^{n}} Q_{i}=\sum_{k=N_{0}+1}^{N} a_{k} \varphi_{\sigma(k)}
$$

Then

$$
\begin{aligned}
\int_{0}^{1}|Q| d t & \leq \sum_{i=1}^{2^{n}} \int_{0}^{1}\left|Q_{i}\right| d t \leq 2 \int_{0}^{1}|f| d t \\
|E| & =\sum_{i=1}^{2^{n}}\left|E_{i}\right|>(1-\varepsilon) \sum_{i=1}^{2^{n}}\left|\Delta_{i}\right|=(1-\varepsilon)|\Delta|
\end{aligned}
$$

and

$$
\delta>\left|a_{k}\right| \geq\left|a_{k+1}\right|>0, \forall k \in\left(N_{0}, N\right) .
$$

Finally, to each $m \in\left(N_{0}, N\right]$ there corresponds a unique $j \in\left[1,2^{n}\right]$ such that $t_{j-1}<m \leq t_{j}$. Thus,

$$
\sum_{k=N_{0}+1}^{m} a_{k} \varphi_{\sigma(k)}=\sum_{i=1}^{j-1} Q_{i}+\sum_{k=t_{j-1}+1}^{m} a_{k} \varphi_{\sigma(k)},
$$

so that

$$
\begin{aligned}
\int_{0}^{1}\left|\sum_{k=N_{0}+1}^{m} a_{k} \varphi_{\sigma(k)}\right| d t & \leq \sum_{i=1}^{j-1} \int_{0}^{1}\left|Q_{i}\right| d t+\int_{0}^{1}\left|\sum_{k=t_{j-1}+1}^{m} a_{k} \varphi_{\sigma(k)}\right| d t \\
& \leq 2 \int_{0}^{1}|f| d t+A\left|\gamma_{j}\right| \varepsilon^{-\frac{1}{2}}\left|\Delta_{j}\right|^{\frac{1}{2}} \\
& <3 \int_{0}^{1}|f| d t .
\end{aligned}
$$

4

The proof of Theorem 2 proceeds along the following lines.

Let $\left\{f_{n}\right\}_{n=1}^{\infty}$ be an enumeration of the set of all step functions defined on dyadic partitions of $[0,1]$ and assuming only rational values.

By virtue of Lemma 4 , one may determine numerical sequences $\left\{\bar{m}_{k}\right\}_{k=0}^{\infty}$ and $\left\{m_{k}\right\}_{k=0}^{\infty}$, with $\bar{m}_{0}=m_{0}=0$, and

$$
m_{k} \leq \bar{m}_{k}<m_{k+1} \leq \bar{m}_{k+1}, \forall k \geq 0
$$


a sequence of measurable subsets of $[0,1],\left\{E_{n}\right\}_{n=1}^{\infty}$, and Walsh polynomials

$$
Q_{n}=\sum_{k=\bar{m}_{n-1}+1}^{m_{n}} a_{k}^{(n)} \varphi_{\sigma_{n}(k)}, n=1,2, \ldots,
$$

where $\sigma_{n}$ is a permutation of $\left\{\bar{m}_{n-1}+1, \ldots, \bar{m}_{n}\right\}$, such that

$$
\begin{gathered}
\left|E_{n}\right|>1-\varepsilon, \\
Q_{n}(x)=f_{n}(x), \forall x \in E_{n}, \\
\int_{0}^{1}\left|Q_{n}\right| d t \leq 2 \int_{0}^{1}\left|f_{n}\right| d t, \\
\int_{0}^{1}\left|\sum_{k=\bar{m}_{n-1}+1}^{m} a_{k} \varphi_{\sigma_{n}(k)}\right| d t<3 \int_{0}^{1}\left|f_{n}\right| d t, \forall m \in\left(\bar{m}_{n-1}, \bar{m}_{n}\right], n=1,2, \ldots, \\
\left|a_{k}^{(n)}\right| \geq\left|a_{k+1}^{(n)}\right|, \forall k \in\left(\bar{m}_{n-1}, \bar{m}_{n}\right), n=1,2, \ldots,
\end{gathered}
$$

and

$$
\left|a_{m_{n}}^{(n)}\right|>\left|a_{k}^{(n+1)}\right|>0, \forall k \in\left(\bar{m}_{n}, m_{n+1}\right), n=1,2, \ldots
$$

Let $t_{0}=0$ and, for $j \in \mathbb{N}$, let $t_{j}=\sum_{i=0}^{j} m_{i}-\sum_{i=0}^{j-1} \bar{m}_{i}$. For $t_{j}<k \leq t_{j+1}$, $j=0,1, \ldots$, let

$$
\sigma(k)=\sigma_{j+1}\left(k+\bar{m}_{j}-t_{j}\right),
$$

let

$$
a_{k}=a_{k+\bar{m}_{j}-t_{j}}^{(j+1)}
$$

and let

$$
E=\bigcap_{n=1}^{\infty} E_{n} .
$$

Then

$$
\begin{aligned}
& Q_{n}=\sum_{k=t_{n-1}+1}^{t_{n}} a_{k} \varphi_{\sigma(k)}, \forall n \in \mathbb{N}, \\
&\left|a_{k}\right| \geq\left|a_{k+1}\right|>0, \forall k \in \mathbb{N},
\end{aligned}
$$

and

$$
|E|>1-\varepsilon .
$$

Now let $f$ be an arbitrary element of $L^{1}[0,1]$. One determines the required approximant to $f$ in the following manner.

Choose $k_{1}$ so that

$$
\left\|f-f_{k_{1}}\right\|_{1}<\frac{1}{8}
$$

and choose $k_{2}>k_{1}$ so that

$$
\left\|f-f_{k_{1}}-f_{k_{2}}\right\|_{1}<\frac{1}{4^{2}} .
$$

Then

$$
\left\|f_{k_{2}}\right\|_{1}<\frac{1}{2^{2}} .
$$


Suppose that the functions $f_{k_{1}}, \ldots, f_{k_{m}}$ have been determined so that $k_{j}<k_{j+1}$, for $j=1, \ldots, n-1$, and for all $m \in[2, n]$, both

$$
\left\|f-\sum_{j=1}^{m} f_{k_{j}}\right\|_{1}<\frac{1}{4^{m}}
$$

and

$$
\left\|f_{k_{m}}\right\|_{1}<\frac{1}{2^{m}}
$$

Choose $k_{n+1}>k_{n}$ so that

$$
\left\|f-\sum_{j=1}^{n} f_{k_{j}}-f_{k_{n+1}}\right\|_{1}<\frac{1}{4^{n+1}} .
$$

Then

$$
\left\|f_{k_{n+1}}\right\|_{1} \leq \frac{1}{4^{n}}+\frac{1}{4^{n+1}}<\frac{1}{2^{n+1}} .
$$

Let $\left\{Q_{k_{n}}\right\}_{n=1}^{\infty}$ be the sequence of Walsh polynomials that corresponds to $\left\{f_{k_{n}}\right\}_{n=1}^{\infty}$, and let

$$
g_{n}=\sum_{j=1}^{n} Q_{k_{j}}
$$

For $m>n$, one has

$$
\left\|g_{m}-g_{n}\right\|_{1} \leq \sum_{j=n+1}^{m}\left\|Q_{k_{j}}\right\|_{1} \leq 2 \sum_{j=n+1}^{m}\left\|f_{k_{j}}\right\|_{1}<\frac{2}{2^{n}} ;
$$

thus, $\left\{g_{n}\right\}_{n=1}^{\infty}$ converges, in the $L^{1}$ norm, to

$$
g=\sum_{n=1}^{\infty} Q_{k_{n}}
$$

Moreover, for all $x$ in $E$, one has

$$
Q_{k_{n}}(x)=f_{k_{n}}(x), \forall n
$$

so that

$$
g(x)=f(x), \text { for a.a. } x \in E .
$$

If one defines the sequence $\left\{\varepsilon_{j}\right\}_{j=1}^{\infty}$ by the conditions

$$
\varepsilon_{j}= \begin{cases}1, & \text { if } t_{k_{n}-1}<j \leq t_{k_{n}} \\ 0, & \text { otherwise }\end{cases}
$$

then the series

$$
\sum_{k=1}^{\infty} \varepsilon_{k} a_{k} \varphi_{\sigma(k)}
$$

converges to $g$, in the $L^{1}$ norm, as the following estimates show.

Let

$$
S_{n}=\sum_{k=1}^{n} \varepsilon_{k} a_{k} \varphi_{\sigma(k)} .
$$

If $\varepsilon_{n}=0$, let $q$ be the unique natural number such that

$$
t_{k_{q}}<n \leq t_{k_{q+1}-1} \text {. }
$$


Then

$$
\left\|S_{n}-g\right\|_{1} \leq \sum_{j=q+1}^{\infty}\left\|Q_{k_{j}}\right\|_{1} \leq 2 \sum_{j=q+1}^{\infty}\left\|f_{k_{j}}\right\|_{1}<\frac{1}{2^{q-1}} .
$$

If $\varepsilon_{n}=1$, let $q$ be the unique natural number such that

$$
t_{k_{q}-1}<n \leq t_{k_{q}}
$$

Then,

$$
\begin{aligned}
\left\|S_{n}-g\right\|_{1} & \leq \sum_{j=q+1}^{\infty}\left\|Q_{k_{j}}\right\|_{1}+\left\|\sum_{k=t_{k_{q}-1}+1}^{n} a_{k} \varphi_{\sigma(k)}\right\|_{1} \\
& \leq 3 \sum_{j=q}^{\infty}\left\|f_{k_{j}}\right\|_{1}<\frac{1}{2^{q-3}} .
\end{aligned}
$$

Finally, from the orthogonality of the Walsh functions, one has

$$
\begin{aligned}
\varepsilon_{k} a_{k} & =\lim _{n} \int_{0}^{1} S_{n} \varphi_{\sigma(k)} d t \\
& =\int_{0}^{1} g \varphi_{\sigma(k)} d t .
\end{aligned}
$$

Thus, the rearrangement of the Walsh-Fourier series of $g$, according to decreasing magnitudes of the expansion coefficients, converges to $g$ in the $L^{1}$-norm, so that the argument above suffices to establish Theorem 1, as well.

\section{REFERENCES}

1. M.G. Grigorian, On the convergence of the greedy algorithm in the $L^{1}$ norm (to appear).

2. M.G. Grigorian and Robert E. Zink, Subsystems of the Walsh orthogonal system whose multiplicative completions are quasibases for $L^{p}[0,1], 1 \leq p<+\infty$, Proc. Amer. Math. Soc. 131(4) (2002), 1137-1149. MR1948105 (2003k:42052)

3. T.W. Körner, Decreasing rearranged Fourier series, J. Fourier Anal. and Appl. 5 (1999), 1-19. MR1682270 (2000c:42006)

4. J.J. Price, A density theorem for Walsh functions, Proc. Amer. Math. Soc. 18 (1967), 209-211. MR.0209760 (35:656)

5. V.N. Temlyakov, Greedy algorithm and m-term approximation, Constructive Approx. 14 (1998), 569-587. MR1646563 (99k:42006)

Department of Physics, Erevan State University, Alex Manoogian Str., 375049 YereVAN, ARMENIA

Department of Mathematics, Purdue University, West Lafayette, Indiana 47907-1968 